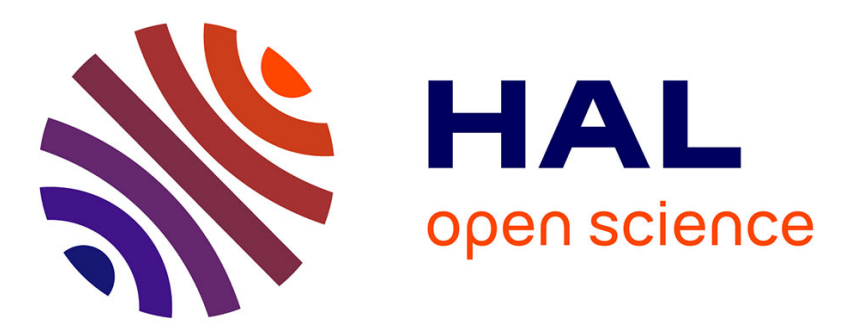

\title{
Switching the Spin on a Ni Trimer within a Metal-Organic Motif by Controlling the On-Top Bromine Atom
}

Lei Xie, Haiping Lin, Chi Zhang, Jingcheng Li, Nestor Merino-Díez, Niklas Friedrich, Xavier Bouju, Youyong Li, Jose Ignacio Pascual, Wei Xu

\section{To cite this version:}

Lei Xie, Haiping Lin, Chi Zhang, Jingcheng Li, Nestor Merino-Díez, et al.. Switching the Spin on a Ni Trimer within a Metal-Organic Motif by Controlling the On-Top Bromine Atom. ACS Nano, 2019, 13 (9), pp.9936-9943. 10.1021/acsnano.9b04715 . hal-02415044

\author{
HAL Id: hal-02415044 \\ https://hal.science/hal-02415044
}

Submitted on 9 Dec 2020

HAL is a multi-disciplinary open access archive for the deposit and dissemination of scientific research documents, whether they are published or not. The documents may come from teaching and research institutions in France or abroad, or from public or private research centers.
L'archive ouverte pluridisciplinaire HAL, est destinée au dépôt et à la diffusion de documents scientifiques de niveau recherche, publiés ou non, émanant des établissements d'enseignement et de recherche français ou étrangers, des laboratoires publics ou privés. 


\section{Switching the Spin on a Ni Trimer within a Metal- Organic Motif by Controlling the On-Top Bromine Atom}

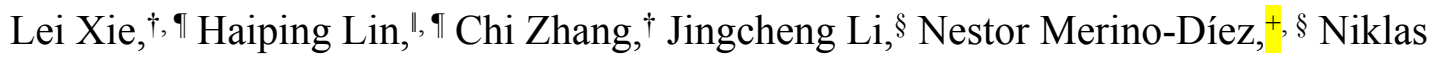
Friedrich, ${ }^{\S}$ Xavier Bouju, ${ }^{\ddagger}$ Youyong Li, Jose Ignacio Pascual, $§, \#, *$ and Wei $\mathrm{Xu}^{\dagger}$,* ${ }^{\dagger}$ Interdisciplinary Materials Research Center, College of Materials Science and Engineering, Tongji University, Shanghai 201804, People's Republic of China

" Institute of Functional Nano \& Soft Materials (FUNSOM), Jiangsu Key Laboratory for CarbonBased Functional Materials \& Devices, Joint International Research Laboratory of Carbon-Based Functional Materials and Devices, Soochow University, 199 Ren'ai Road, Suzhou, 215123, Jiangsu, People's Republic of China

$\S$ CIC nanoGUNE, 20018 San Sebastián-Donostia, Spain

${ }^{+}$Donostia International Physics Center (DIPC), 20018, San Sebastián-Donostia, Spain

$\$$ CEMES-CNRS, Université de Toulouse, Toulouse, France

\# Ikerbasque, Basque Foundation for Science, 48013 Bilbao, Spain

*E-mail: xuwei@tongji.edu.cn, ji.pascual@,nanogune.eu

\section{ABSTRACT}

Controlling the spin of metal atoms embedded in molecular systems is a key step towards the realization of molecular electronics and spintronics. Many efforts have been devoted to explore the influencing factors dictating the survival or quenching of a magnetic moment in a metal-organic molecule, and among others, the spin control by axial ligand attachments is most promising. Herein, from the interplay of highresolution STM imaging/manipulation and STS measurements together with DFT calculations, we successfully demonstrate that a Ni trimer within a metal-organic motif acquires a net spin promoted by the adsorption of an on-top $\mathrm{Br}$ atom. The spin localization in the tri-metal centers bonded to $\mathrm{Br}$ was monitored via the Kondo effect. The removal of the Br ligand resulted in the switch from a Kondo ON to a Kondo OFF state. The magnetic state induced by the Br ligand is theoretically attributed to the enhanced $\mathrm{Br} 4 \mathrm{p}_{\mathrm{z}}$ and $\mathrm{Ni} 4 \mathrm{dz}^{2}$ states due to the charge redistribution. The manipulation strategy reported here provides the possibility to explore potential applications of spin- 
tunable structures in spintronic devices.

KEYWORDS: scanning tunneling microscopy, scanning tunneling spectroscopy, Kondo effect, spin switching, ligand attachment, density functional theory

Controlling the charge and spin at single molecular scale is a key step towards the realization of molecular electronics and spintronics. ${ }^{1-5}$ When a molecular spin lies in the proximity of a metal substrate, it may exhibit the Kondo effect, ${ }^{6,7}$ originating from the screening of the localized spin by conducting electrons. This fundamental effect provides an ideal workbench for identifying magnetic atomic and molecular species on surfaces,${ }^{8,9}$ in break junctions, ${ }^{10,11}$ as well as in single-electron transistors. ${ }^{12-17}$ Its detection has been used as a fingerprint of paramagnetism in systems such as in $\mathrm{C}_{60},{ }^{17}$ carbon nanotubes, ${ }^{18}$ graphene ${ }^{19}$ and different edge states of graphene nanoribbons ${ }^{20}$ obtained by on-surface synthesis. Scanning tunneling microscopy has been proven to be a powerful tool in the characterization of the spin state in magnetic systems. ${ }^{21-27}$ Many efforts have been devoted to understanding the influencing factors behind the survival of a molecular spin in contact with a metal surface ${ }^{28}$ and determining basic rules for their manipulation. For example, numerous studies of organo-metallic species with a single metal center, such as metallo-porphyrins and -phthalocyanines molecules, reported that the molecular spin can be manipulated by tuning the periphery chemical environment, ${ }^{29-33}$ conformational change, ${ }^{34-36}$ the adsorption site on a substrate, ${ }^{37-39}$ the structure of the self-assembled domain, ${ }^{35,40,41}$ and the axial coordination ${ }^{5,42-46}$. In particular, the possibility of modifying the Kondo state of a magnetic molecule by changing its axial ligand coordination with the attachment of small molecules such as atomic hydrogen, ${ }^{5} \mathrm{Cl},{ }^{42} \mathrm{CO},{ }^{43},{ }^{44} \mathrm{NO},{ }^{43} \mathrm{NH}_{3},{ }^{45}$ and alkali metals ${ }^{46}$ attracts a lot of attentions due to the potential applications as gas sensors. However, the study of the magnetic ground state of multiple metal centers into complex assemblies is still elusive, as well as the development of possible manipulation routes that could enable/disable their Kondo screening with the substrate. These would entitle the design of novel devices for electrical sensing chemical signals. 
In this study, we report on the manipulation of the magnetic state of a multiple-metal center in a molecular assembly by addition/removal of $\mathrm{Br}$ atoms. Based on our previous study, ${ }^{47}$ we chose a derivative of the guanine molecule (9-ethylguanine, abbreviated as G) and a $\mathrm{NiBr}_{2}$ salt with the aim of the fabricating a three-metal-center (i.e., Ni trimer, shortened as $\mathrm{Ni}_{3}$ ) metal-organic system on the $\mathrm{Au}(111)$ surface. Through a combination of high-resolution scanning tunneling microscopy (STM), scanning tunneling spectroscopy (STS), density functional theory (DFT) calculations and STM image simulations, we demonstrate that this system shows an intermediate configuration with a $\mathrm{Br}$ atom sitting on top of the hollow site of the central $\mathrm{Ni}_{3}$ cluster, which modifies the electronic configuration of the three-metal center. Annealing to higher temperatures, the $\mathrm{G}_{3} \mathrm{Ni}_{3} \mathrm{Br}_{4}$ metal-organic network is transformed into $\mathrm{G}_{3} \mathrm{Ni}_{3} \mathrm{Br}_{3}$, where the on-top $\mathrm{Br}$ atom is vanished. Moreover, in-situ removal of the on-top $\mathrm{Br}$ atom by STM manipulation is also achieved. Differential conductance $(\mathrm{dI} / \mathrm{dV})$ spectra on the $\mathrm{G}_{3} \mathrm{Ni}_{3} \mathrm{Br}_{4}$ structures show a pronounced zero-bias peak at Fermi level assigned to the signature of Kondo effect, which is absent on the $\mathrm{G}_{3} \mathrm{Ni}_{3} \mathrm{Br}_{3}$ structures (cf. Scheme 1); thus, removal of on-top $\mathrm{Br}$ atoms of the $\mathrm{G}_{3} \mathrm{Ni}_{3} \mathrm{Br}_{4}$ structure through annealing or in-situ STM manipulation removes the zero-energy Kondo resonance. Such an on-top $\mathrm{Br}$ adsorption induced Kondo effect on a $\mathrm{Ni}_{3}$ cluster within a metal-organic structure is theoretically interpreted by a charge redistribution over $d$ orbitals of Ni leading to the gain of a net magnetization. 
Kondo ON

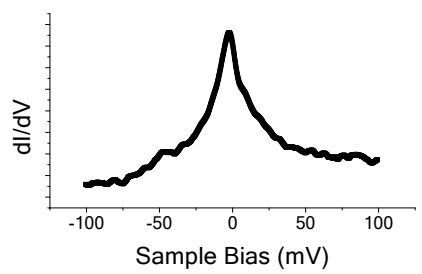

Kondo OFF

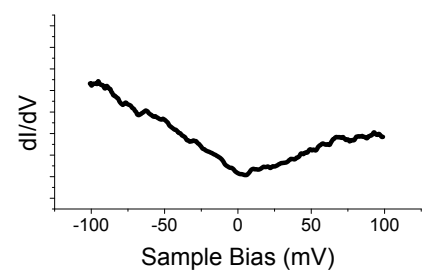

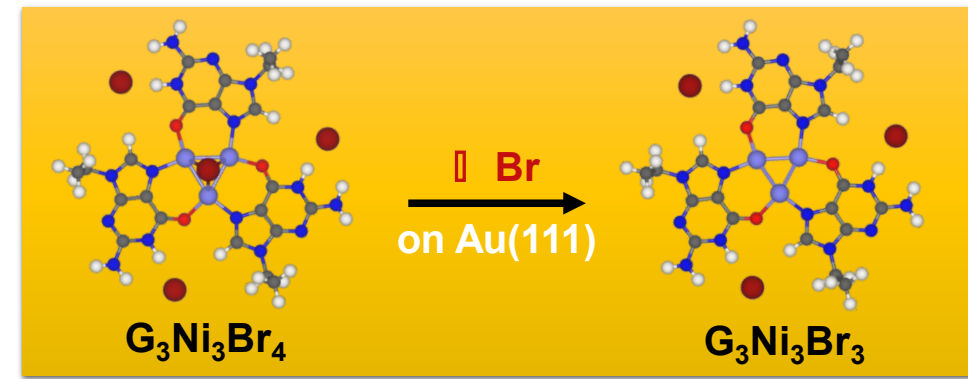

Scheme 1. Schematic illustration of the on-top $\mathrm{Br}$ atom induced Kondo effect on a three-metal center within a metal-organic structure. Kondo effect is generated by the attachment of a $\mathrm{Br}$ atom above the $\mathrm{Ni}_{3}$ cluster within the $\mathrm{G}_{3} \mathrm{Ni}_{3} \mathrm{Br}_{4}$ motif on the $\mathrm{Au}(111)$ surface. After annealing or STM manipulation, the on-top Br atom is removed and the Kondo effect vanishes in the resulted $\mathrm{G}_{3} \mathrm{Ni}_{3} \mathrm{Br}_{3}$ motif.

\section{RESULTS AND DISCUSSION}

The co-deposition of $\mathrm{G}$ molecules and $\mathrm{NiBr}_{2}$ on the $\mathrm{Au}(111)$ surface held at room temperature (RT) results in close-packed islands like the one shown in Figure 1a. A close-up STM image allows us to identify the elementary structural motifs, depicted by white contours in Figure 1b. The characteristic motifs (Figure 1c) are composed of a bright dot at the center, assigned to an on-top $\mathrm{Br}$ atom, and six peripheral protrusions around the triangular base. ${ }^{42}$ This assignment is also confirmed by the Br removal experiment discussed below. The structural motif beneath the central $\mathrm{Br}$ atom coincides in size and shape with the structure of the basic motif of $\mathrm{G}_{3} \mathrm{Ni}_{3} \mathrm{I}_{3}$ assemblies, reported previously. ${ }^{47}$ Thus, we tentatively assign the $\mathrm{G}_{3} \mathrm{Ni}_{3} \mathrm{Br}_{4}$ motif (Figure 1c) to a structure formed by $\mathrm{a}_{3} \mathrm{Ni}_{3} \mathrm{Br}_{3}$ base with an additional $\mathrm{Br}$ atom sitting on the hollow site of the central $\mathrm{Ni}_{3}$ cluster. This structure is successfully reproduced by DFT structural simulations for $\mathrm{a}_{3} \mathrm{Ni}_{3} \mathrm{Br}_{4}$ cluster on the $\mathrm{Au}(111)$ surface, resulting in the optimized structural model superimposed on the STM image in Figure 1c, and depicted in Figure 
1d. According to this, the motif is composed of three homochiral $G$ molecules coordinated with three $\mathrm{Ni}$ atoms located at the hollow sites of the $\mathrm{Au}(111)$ surface. Three peripheral $\mathrm{Br}$ atoms are uniformly located at the specific hydrogen-rich harbors via electrostatic interactions. Importantly, the top $\mathrm{Br}$ atom sits on top of the hollow site of the $\mathrm{Ni}_{3}$ cluster, with an adsorption energy of $-1.40 \mathrm{eV}$. The corresponding ESQC calculated STM image, shown in Figure 1e, reproduces the major details of our STM images with a large central spot corresponding to the $\mathrm{Br}-\mathrm{Ni}_{3}$ motif, which is brighter than both the lateral ethyl groups and the on-surface $\mathrm{Br}$ atoms. The $\mathrm{G}_{3} \mathrm{Ni}_{3} \mathrm{Br}_{4}$ motifs are then linked together by hydrogen bonds and van der Waals interactions to form extended metal-organic islands, as shown in Figure 1f.
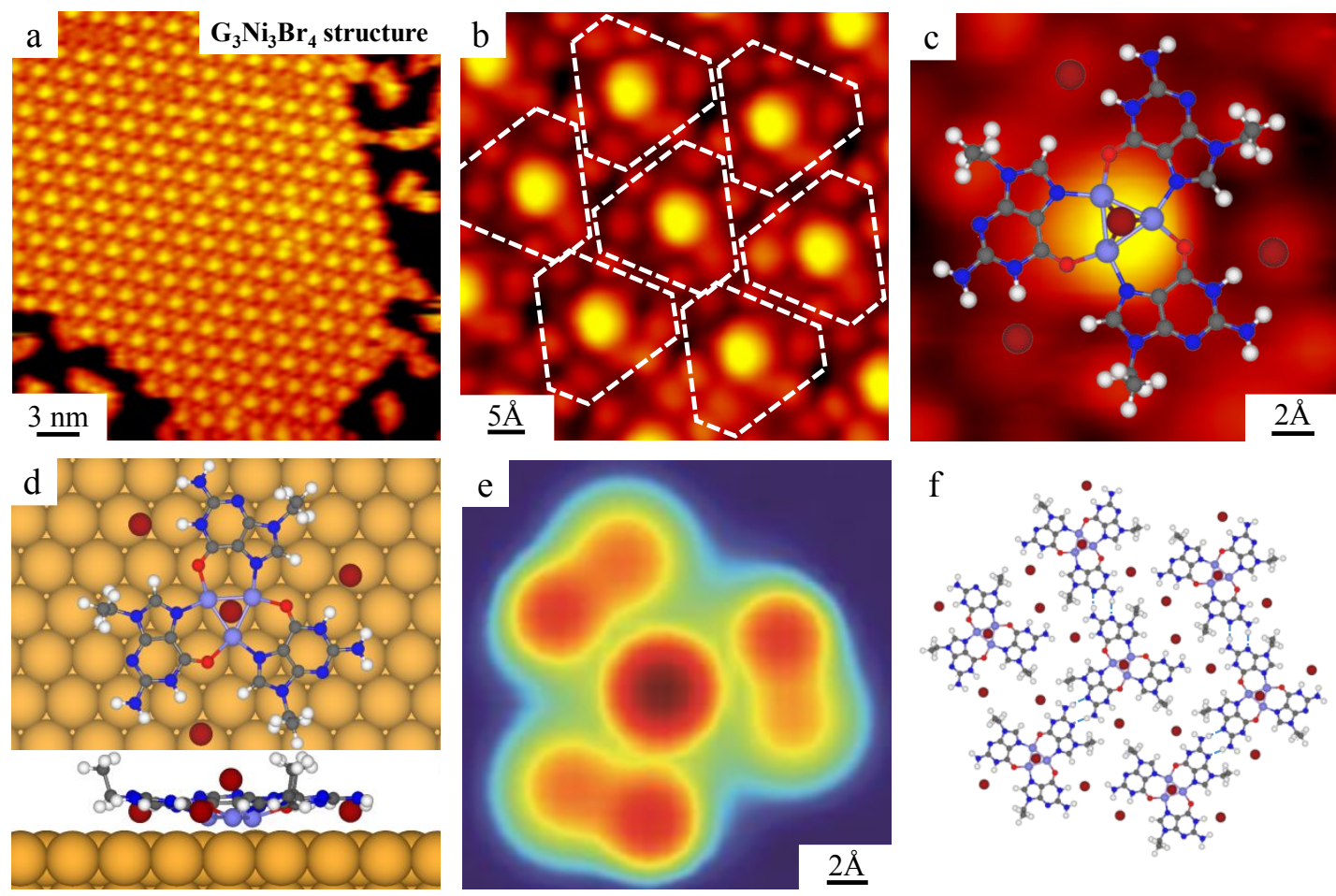

Figure 1. Formation of the $\mathrm{G}_{3} \mathrm{Ni}_{3} \mathrm{Br}_{4}$ network structure after the deposition of $\mathrm{G}$ molecules and $\mathrm{NiBr}_{2}$ on $\mathrm{Au}(111)$ held at RT. (a) Large-scale STM image showing the close-packed island composed of the $\mathrm{G}_{3} \mathrm{Ni}_{3} \mathrm{Br}_{4}$ structure. (b) Close-up STM image showing the details of the structure in which $\mathrm{G}_{3} \mathrm{Ni}_{3} \mathrm{Br}_{4}$ structures are indicated by white contours. (c) A further zoomed-in STM image of the $\mathrm{G}_{3} \mathrm{Ni}_{3} \mathrm{Br}_{4}$ motif superimposed with the DFT-optimized gas-phase model. (d) Top and side views of the DFT-relaxed model of the $\mathrm{G}_{3} \mathrm{Ni}_{3} \mathrm{Br}_{4}$ motif on $\mathrm{Au}(111)$. H: white, $\mathrm{C}$ : gray, $\mathrm{N}$ : blue, O: red, Ni: light blue, $\mathrm{Br}$ : 
brown, Au: yellow. (e) The ESQC calculated STM image of the $\mathrm{G}_{3} \mathrm{Ni}_{3} \mathrm{Br}_{4}$ motif at $\mathrm{E}_{\mathrm{f}}$. (f) DFT-optimized gas-phase model of the $\mathrm{G}_{3} \mathrm{Ni}_{3} \mathrm{Br}_{4}$ network structure. Hydrogen bonds are depicted by blue dashed lines.

As we will show next, the axial attachment of a $\mathrm{Br}$ atom on top of the $\mathrm{Ni}_{3}$ cluster has important consequences on their electronic configuration. First, STS measurements uncover a net magnetization of the $\mathrm{G}_{3} \mathrm{Ni}_{3} \mathrm{Br}_{4}$ metal organic moiety. Figure $2 \mathrm{~b}$ shows a representative $\mathrm{dI} / \mathrm{dV}$ point spectrum taken on-top of the central $\mathrm{Br}$ atom ( $c f$. the black cross in Figure 2a), which shows a pronounced, narrow peak centered at zero bias. Based on both the bias position and the logarithmic-like shape of the peak we attribute it to an Abrikosov-Suhl resonance due to the Kondo effect, ${ }^{21}$ and named as Kondo resonances herein. The coupling between the magnetic center and surrounding free electrons has further been quantified by fitting the Kondo resonance with a Fano function ${ }^{48,49}$ (see details in the Supporting Information), obtaining a Kondo temperature of $\mathrm{T}_{\mathrm{K}} \sim 124 \mathrm{~K}$. The extension of the Kondo signal in STM experiments has been related to the spin localization. ${ }^{50,51}$ Accordingly, we conducted $20 \mathrm{dI} / \mathrm{dV}$ point spectra along a line between two adjacent on-top $\mathrm{Br}$ atoms (black line indicated in Figure 2a). The resulting spectral map along the line (shown as a vertical axis in Figure 2c, with the colors representing the $\mathrm{dI} / \mathrm{dV}$ intensity) shows that the zero-bias resonances are localized within the range of the metal centers. Furthermore, these resonances are unaffected by the proximity of edges or defects, suggesting that the coupling between neighboring metal-organic motifs is negligibly small. ${ }^{41}$
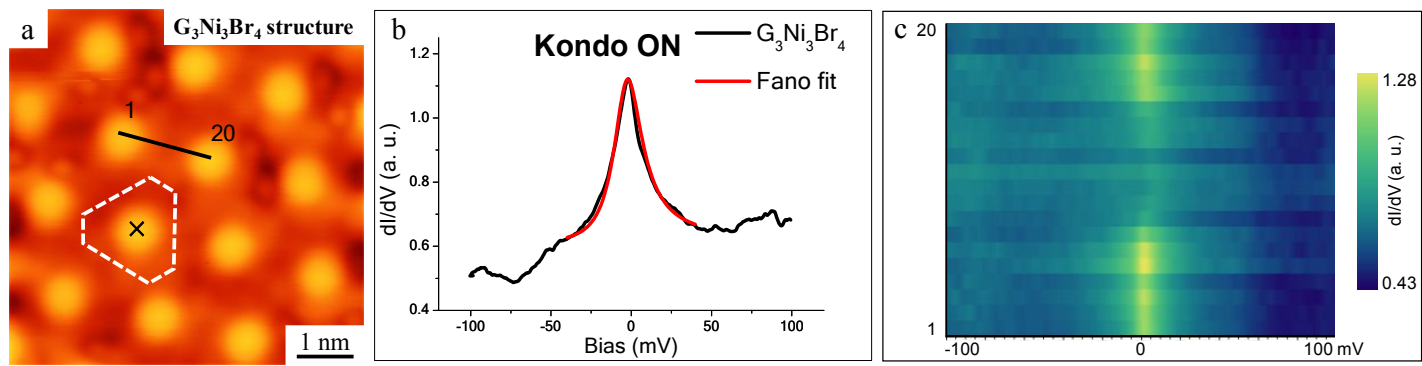

Figure 2. Characterization of the electronic properties of the $\mathrm{G}_{3} \mathrm{Ni}_{3} \mathrm{Br}_{4}$ structure. (a) An STM image with a $\mathrm{G}_{3} \mathrm{Ni}_{3} \mathrm{Br}_{4}$ motif highlighted by the white contour. (b) A 
representative experimental $\mathrm{dI} / \mathrm{dV}$ point spectrum (the black curve) obtained at the black cross shown in (a), which shows a pronounced peak at the Fermi level representing the signature of Kondo effect (open-feedback parameters: $\mathrm{V}_{\mathrm{t}}=1.0 \mathrm{~V}, \mathrm{I}_{\mathrm{t}}=$ $1 \mathrm{nA}$, modulation voltage $\mathrm{V}_{\mathrm{rms}}=0.4 \mathrm{mV}$, lock-in frequency: $760 \mathrm{~Hz}$ ); the corresponding Fano fit curve (the red one) $(\mathrm{q}=10, \Gamma=10.8 \mathrm{mV})$ shows a good agreement. The flat density of states of the STM tip is checked before and after on the bare Au(111) surface, where it only displays the well-known surface state onset (cf. Figure S1). (c) The spectral map of 20 spectra taken along the black line in (a) stacked vertically with colors corresponding to $\mathrm{dI} / \mathrm{dV}$ intensity.

After a thermal treatment of the sample at $390 \mathrm{~K}$ for $10 \mathrm{~min}$, a honeycomb network like the one shown in Figure 3a is observed instead. Close-up STM images resolve that these structures are formed by the elementary triangular motifs, depicted by white contours in Figure 3b. These motifs are assigned to $\mathrm{G}_{3} \mathrm{Ni}_{3} \mathrm{Br}_{3}$ clusters, according to the identical configuration of $\mathrm{G}_{3} \mathrm{Ni}_{3} \mathrm{I}_{3}$ honeycomb network structure in our previous study. ${ }^{47}$ Also note that this structure coincides with the $\mathrm{G}_{3} \mathrm{Ni}_{3} \mathrm{Br}_{4}$ motif from above, but with the ontop $\mathrm{Br}$ atoms removed probably due to the annealing process. A further zoomed-in STM image superimposed with the DFT-optimized gas-phase model is shown in Figure 3c. It is noticeable that the electronic density of states of the $\mathrm{Ni}_{3}$ cluster within the $\mathrm{G}_{3} \mathrm{Ni}_{3} \mathrm{Br}_{3}$ motif is less pronounced in comparison with the case of $\mathrm{G}_{3} \mathrm{Ni}_{3} \mathrm{I}_{3}$. The top and side views of the DFT optimized structural model of $\mathrm{G}_{3} \mathrm{Ni}_{3} \mathrm{Br}_{3}$ on $\mathrm{Au}(111)$ are shown in Figure $3 \mathrm{~d}$. We find that here the $\mathrm{Ni}_{3}$ cluster lies $0.3 \AA$ closer to the $\mathrm{Au}(111)$ surface than in the $\mathrm{G}_{3} \mathrm{Ni}_{3} \mathrm{Br}_{4}$ motif, presumably suffering a larger hybridization with metal states. The corresponding ESQC calculated STM image is shown in Figure 3e, assigning the peripheral bright spots to ethyl groups pointing upwards and to the close-by $\mathrm{Br}$ adatoms. The elementary $\mathrm{G}_{3} \mathrm{Ni}_{3} \mathrm{Br}_{3}$ motifs further interact with each other by double hydrogen bonds resulting in the formation of the honeycomb network shown in Figure $3 \mathrm{f}$. 

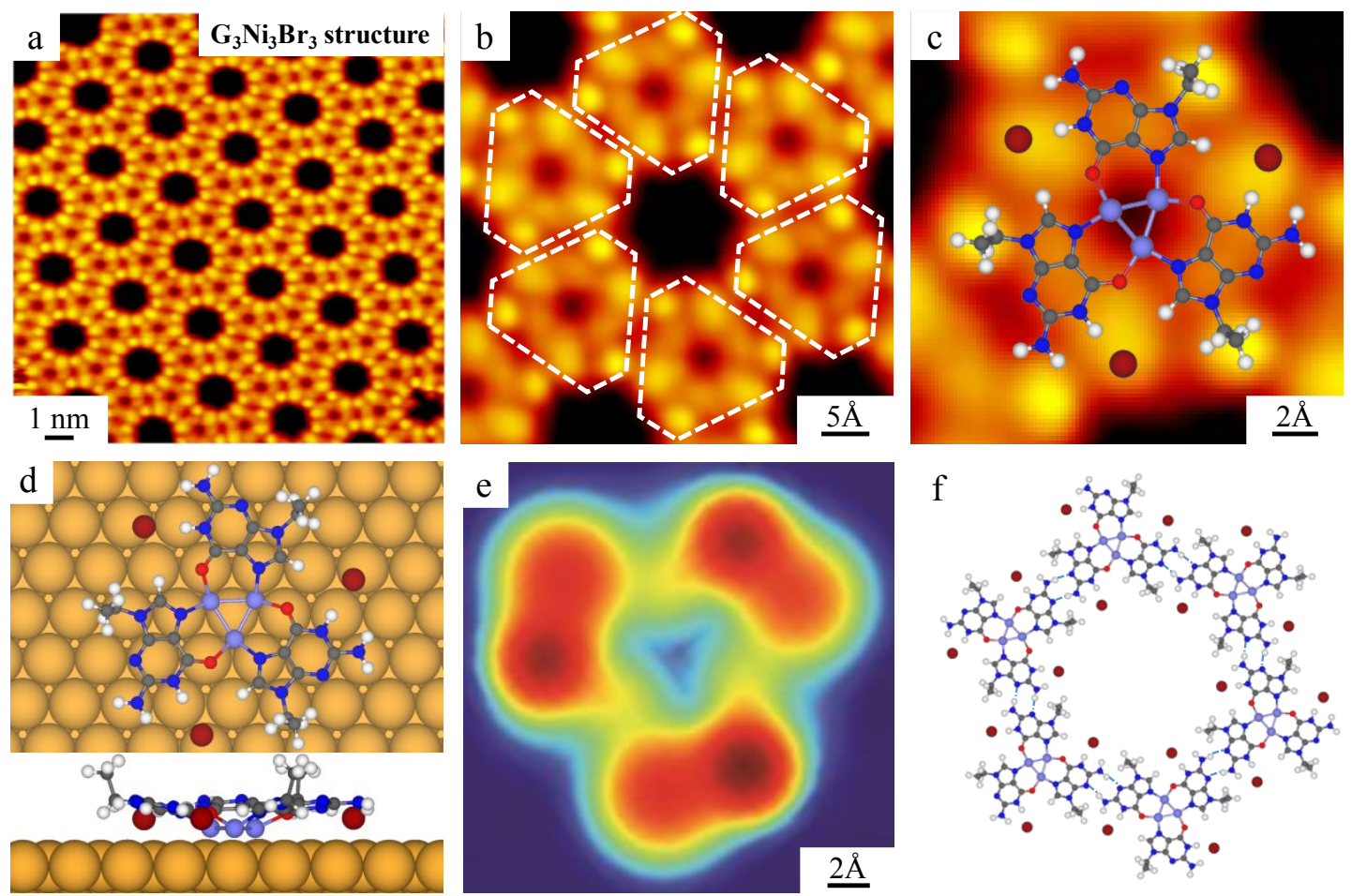

Figure 3. Formation of the $\mathrm{G}_{3} \mathrm{Ni}_{3} \mathrm{Br}_{3}$ network structure after annealing the sample of $\mathrm{G}_{3} \mathrm{Ni}_{3} \mathrm{Br}_{4}$ phase at $390 \mathrm{~K}$ on $\mathrm{Au}(111)$. (a) Large-scale $\mathrm{STM}$ image showing the honeycomb network composed of the $\mathrm{G}_{3} \mathrm{Ni}_{3} \mathrm{Br}_{3}$ structure. (b) Close-up STM image showing the details of the structure in which $\mathrm{G}_{3} \mathrm{Ni}_{3} \mathrm{Br}_{3}$ structures are indicated by white contours. (c) A further zoomed-in STM image of the $\mathrm{G}_{3} \mathrm{Ni}_{3} \mathrm{Br}_{3}$ motif superimposed with the DFT-optimized gas-phase model. (d) Top and side views of the DFT-relaxed model of the $\mathrm{G}_{3} \mathrm{Ni}_{3} \mathrm{Br}_{3}$ motif on $\mathrm{Au}(111)$. (e) The ESQC calculated STM image of the $\mathrm{G}_{3} \mathrm{Ni}_{3} \mathrm{Br}_{3}$ motif at $\mathrm{E}_{\mathrm{f}}$. (f) DFT-optimized gas-phase model of the $\mathrm{G}_{3} \mathrm{Ni}_{3} \mathrm{Br}_{3}$ network structure.

In contrast to $\mathrm{G}_{3} \mathrm{Ni}_{3} \mathrm{Br}_{4}, \mathrm{dI} / \mathrm{dV}$ spectra obtained at the center of the $\mathrm{G}_{3} \mathrm{Ni}_{3} \mathrm{Br}_{3}$ motif (i.e., the spectrum in Figure $4 \mathrm{~b}$ taken over the black cross in Figure 4a) show a relatively featureless curve near the zero bias ( $c f$. Figure $4 \mathrm{~b}$ ), which indicates the absence of a Kondo effect upon removal of the on-top $\mathrm{Br}$ atom. These results suggest that the magnetic moment concluded after the Kondo resonance in $\mathrm{G}_{3} \mathrm{Ni}_{3} \mathrm{Br}_{4}$ clusters is induced by the presence of the $\mathrm{Br}$ atom on top of the $\mathrm{Ni}_{3}$ moiety and vanishes when this halogen atom is removed. By delicately controlling the annealing procedure (at $\sim 380 \mathrm{~K}$ for 10 min), an intermediate state composed of both $\mathrm{G}_{3} \mathrm{Ni}_{3} \mathrm{Br}_{4}$ and $\mathrm{G}_{3} \mathrm{Ni}_{3} \mathrm{Br}_{3}$ motifs can be 
obtained (shown in Figure S2). In this mixed state, dI/dV spectra (Figure S3) further corroborated the coexistence of both magnetic ground states (Kondo ON or OFF) depending on the presence of the $\mathrm{Br}$ atom on top. This suggests that the triangular motifs remain at the annealing temperatures, and that the only transformation consists on the detachment of the $\mathrm{Br}$ atom from the central part of the moiety.

These results suggest that the Kondo state of a molecular $\mathrm{G}_{3} \mathrm{Ni}_{3} \mathrm{Br}_{4}$ cluster could be switched OFF by means of atomic-scale manipulation experiments using the STM. As shown in Figure 4c and 4d, the STM tip was used to remove the on-top Br atom from a $\mathrm{G}_{3} \mathrm{Ni}_{3} \mathrm{Br}_{4}$ motif resulting in the formation of the $\mathrm{G}_{3} \mathrm{Ni}_{3} \mathrm{Br}_{3}$ one. Correspondingly, the characteristic Kondo peak (red curve in Figure 4e) of a $\mathrm{G}_{3} \mathrm{Ni}_{3} \mathrm{Br}_{4}$ motif, which is acquired before the manipulation on the species marked in Figure 4c, disappears after removing the on-top $\mathrm{Br}$ atom ( $c f$. blue curve acquired at the center of the resulting $\mathrm{G}_{3} \mathrm{Ni}_{3} \mathrm{Br}_{3}$ motif in Figure $4 d$ ).

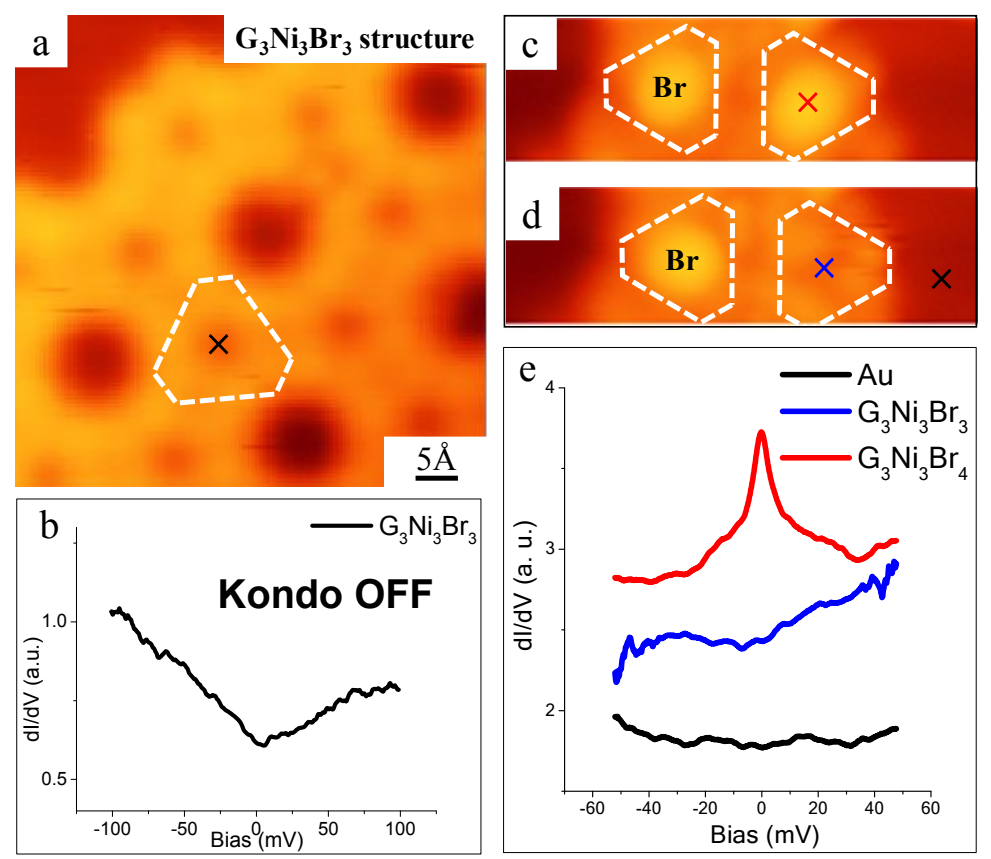

Figure 4. Characterization of the electronic properties of the $\mathrm{G}_{3} \mathrm{Ni}_{3} \mathrm{Br}_{3}$ structure. (a) An STM image with a $\mathrm{G}_{3} \mathrm{Ni}_{3} \mathrm{Br}_{3}$ motif highlighted by the white contour. (b) A representative experimental $\mathrm{dI} / \mathrm{dV}$ point spectrum obtained at the black cross shown in (a), which shows the vanishment of the pronounced Kondo peak. (c) and (d) An in-situ 
STM manipulation to remove the on-top $\mathrm{Br}$ atom in the $\mathrm{G}_{3} \mathrm{Ni}_{3} \mathrm{Br}_{4}$ motif (by a bias ramping to $\sim 3.2 \mathrm{~V}$ ). (e) The $\mathrm{dI} / \mathrm{dV}$ spectra acquired at three points, respectively, that is, with the $\mathrm{Br}$ atom on top (red), without the $\mathrm{Br}$ atom (blue), and on the bare surface (black) (open-feedback parameters: $\mathrm{V}_{\mathrm{t}}=1.0 \mathrm{~V}, \mathrm{I}_{\mathrm{t}}=1 \mathrm{nA}$, modulation voltage $\mathrm{V}_{\mathrm{rms}}=0.4 \mathrm{mV}$, lock-in frequency: $760 \mathrm{~Hz}$ ).

To understand the underlying mechanism behind the formation of a net magnetic moment induced by the on-top $\mathrm{Br}$-atom, and its disappearance on the $\mathrm{G}_{3} \mathrm{Ni}_{3} \mathrm{Br}_{3}$ motifs, extensive DFT calculations have been performed. The incorporation of a $\mathrm{Br}$ atom ontop of a $\mathrm{G}_{3} \mathrm{Ni}_{3} \mathrm{Br}_{3}$ cluster is accompanied by a net energy gain of $1.4 \mathrm{eV}$. The $\mathrm{Br}$ atom binds to the three Ni atoms and slightly pulls them upwards away from the surface by $0.3 \AA$, thus reducing their coupling with substrate states. This distortion induces a charge redistribution and builds up a net magnetic moment in the cluster. In Figure 5a and $b$, the spin-densities of the $\mathrm{G}_{3} \mathrm{Ni}_{3} \mathrm{Br}_{3}$ and $\mathrm{G}_{3} \mathrm{Ni}_{3} \mathrm{Br}_{4}$ motifs are depicted, respectively. The spin-up and spin-down states (purple and green contours) are uniformly distributed near the $\mathrm{Ni}_{3}$ cluster and the $\mathrm{Au}(111)$ surface in the $\mathrm{G}_{3} \mathrm{Ni}_{3} \mathrm{Br}_{3}$ motif, while in $\mathrm{G}_{3} \mathrm{Ni}_{3} \mathrm{Br}_{4}$ clusters the spin-up state is significantly more pronounced near the top $\mathrm{Br}$ atom. Further analysis shows that on the $\mathrm{Au}(111)$ surface, the magnetic moments of the $\mathrm{G}_{3} \mathrm{Ni}_{3} \mathrm{Br}_{3}$ and $\mathrm{G}_{3} \mathrm{Ni}_{3} \mathrm{Br}_{4}$ motifs are $-0.05 \mu \mathrm{B}$ and $0.88 \mu \mathrm{B}$, respectively. The spin polarized density of states (SPDOS) on the $4 \mathrm{~d}$ states of the $\mathrm{Ni}_{3}$ cluster show that the $4 \mathrm{dz}^{2}$ states in the $\mathrm{G}_{3} \mathrm{Ni}_{3} \mathrm{Br}_{4}$ clearly shift upwards, partially crossing the Fermi level (cf. Figure 5d) in comparison with that in the $\mathrm{G}_{3} \mathrm{Ni}_{3} \mathrm{Br}_{3}(c f$. Figure $5 \mathrm{c}$ ). Thus, the attachment of a $\mathrm{Br}$ atom on-top induces a charge redistribution described by a partial depopulation of $\mathrm{dz}^{2}$ electrons, which are transferred towards $\mathrm{Br}$ states. In fact, the SPDOS on the p orbitals of the on-top Br atom shows a spin-polarized state as well (cf. Figure S4a, where the bonding configuration between top $\mathrm{Br}$ and $\mathrm{Ni}$ trimer is also described). Further charge differential analysis also indicates that the on-top $\mathrm{Br}$ atom bonds to the $\mathrm{Ni}_{3}$ cluster with the $\mathrm{Br} 4 \mathrm{p}_{\mathrm{z}}$ orbital (Figure $\mathrm{S} 4 \mathrm{~b}$ ). Thus, the enhanced $\mathrm{Br} 4 \mathrm{p}_{\mathrm{z}}$ and $\mathrm{Ni} 4 \mathrm{dz}^{2}$ states due to the formation of $\mathrm{Br}-\mathrm{Ni}$ bonds may be the origin of the apparition of a net magnetic moment, explaining the observation of a Kondo resonance in our experiment. 

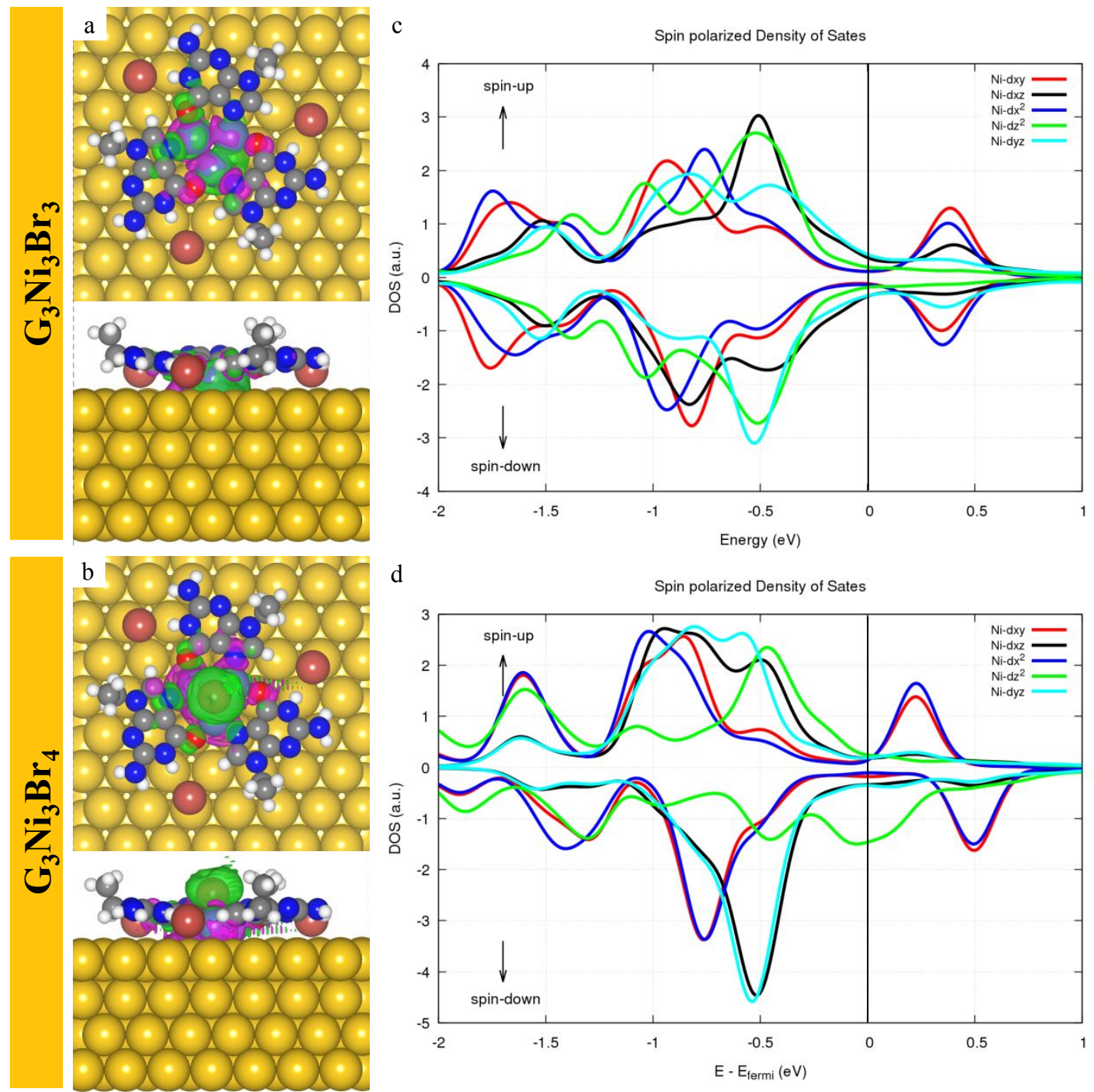

Figure 5. The top and side views of the spin-density of (a) $\mathrm{G}_{3} \mathrm{Ni}_{3} \mathrm{Br}_{3}$ and (b) $\mathrm{G}_{3} \mathrm{Ni}_{3} \mathrm{Br}_{4}$ motifs on the $\mathrm{Au}(111)$ surface. The spin-up and spin-down states are drawn as purple and green contours, respectively. The value of isosurfaces are $\pm 1 \times 10^{-4} \mathrm{e} / \AA^{3}$. The SPDOS of the $4 d$ states of the $\mathrm{Ni}_{3}$ cluster within the (c) $\mathrm{G}_{3} \mathrm{Ni}_{3} \mathrm{Br}_{3}$ and (d) $\mathrm{G}_{3} \mathrm{Ni}_{3} \mathrm{Br}_{4}$ motifs.

\section{CONCLUSIONS}

In summary, here we presented an STM manipulation strategy for controlling the spin of the metallic clusters embedded in a metal-organic structure consisting in the attachment of halogen atoms on top. From a combination of high-resolution STM imaging/manipulation and STS measurements, we have demonstrated that the metal- 
organic motifs containing Ni trimers lies in a magnetic ground state, and exhibits a zerobias Kondo resonance. Comparing with a related structure appearing at higher temperatures with no magnetic fingerprint, we deduce that the magnetic state is promoted by the presence of a $\mathrm{Br}$ atom on top of the Ni trimer. Controllable removal of the on-top $\mathrm{Br}$ atom leads to the disappearance of the magnetic fingerprint from the spectra. Such an experimentally observed Kondo ON state is theoretically attributed to the enhanced $\mathrm{Br} 4 \mathrm{p}_{\mathrm{z}}$ and $\mathrm{Ni} 4 \mathrm{dz}^{2}$ states due to the charge redistribution upon the on-top $\mathrm{Br}$ adsorption. The finding of the magnetic state for Ni trimer could be the basis for the bottom-up assembly of high-spin systems, stabilized by a metal-organic framework (MOF) structure. The spin-manipulation strategy presented here provides the possibility to explore the halogen-driven spin switch in more generalized situations. Utilizing such coordinative interactions of axial halogen atoms to manipulate spin states opens the door to designing spin-tunable interfaces composed of well-defined metalorganic structures with potential applications for spintronic devices.

\section{METHODS}

The $\mathrm{Au}(111)$ substrate was prepared by several cycles of $1.5 \mathrm{keV} \mathrm{Ar}^{+}$sputtering followed by annealing at $820 \mathrm{~K}$ for $15 \mathrm{~min}$, resulting in clean and flat terraces separated by monatomic steps. The $9 \mathrm{eG}$ molecules (purchased from Sigma-Aldrich, purity $>98 \%$ ) and salt $\mathrm{NiBr}_{2}$ (purchased from Sigma-Aldrich, purity $>99 \%$ ) were loaded into separate cells of the crucible in the molecular evaporator oriented to the substrate. After a thorough degassing, the molecules and salt were deposited onto the $\mathrm{Au}(111)$ substrate by thermal sublimation at $440 \mathrm{~K}$ and at $580 \mathrm{~K}$, respectively. The sample was thereafter transferred within the UHV chamber to the STM.

STM images in Figure 1, Figure 3, Figure S2 were acquired in a UHV chamber (base pressure $1 \times 10^{-10}$ mbar) equipped with a variable-temperature, fast scanning "Aarhustype" STM using electrochemically etched W tips purchased from SPECS ${ }^{52,53}$ where measurements were carried out at $\sim 100-150 \mathrm{~K}$. Scanning conditions: $\mathrm{I}_{\mathrm{t}}=0.5 \sim 0.8 \mathrm{nA}$, $\mathrm{V}_{\mathrm{t}}=\sim 1200 \mathrm{mV}$. The other STM images and all spectra are taken on a home-built, lowtemperature STM under UHV at pressures $\sim 1 \times 10^{-11}$ mbar and a base temperature of 
$4.8 \mathrm{~K}$. Scanning conditions: $\mathrm{I}_{\mathrm{t}}=20 \mathrm{pA}, \mathrm{V}_{\mathrm{t}}=\sim 1000 \mathrm{mV}$. All of the STM images were further smoothed to eliminate noises. The $\mathrm{dI} / \mathrm{dV}$ measurements were acquired using lock-in amplifier technique. Analysis of STS data was performed with Origin and SpectraFox software packages. ${ }^{54}$

The structural optimizations and electronic structures of all systems were obtained with the spin-polarized DFT calculations by using the Vienna Ab initio Simulation Package (VASP). ${ }^{55}$ The electron-ion interactions were described with the Projector AugmentedWave (PAW) potentials. ${ }^{56}$ The exchange-correlation interactions of electrons were taken account using the Perdew-Burke-Enzerhof (PBE) of Generalized Gradient Approximation (GGA) pseudopotentials. ${ }^{57}$ The van der Waals interactions were described with the vdW-D3 method. ${ }^{58}$ The cutoff energy of plane wave basis was set as $400 \mathrm{eV}$. The gold surfaces were modelled with a periodic slab consisting of four atomic Au layers, in which the bottom two layers were fixed. All other atoms were optimized until the atomic force was less than $0.01 \mathrm{eV}$ per Angstrom. A vacuum layer of $20 \AA$ was used to avoid the periodic image interactions. The Brillouin zone of reciprocal space was modeled based on the $\Gamma$ centered Monkhorst-Pack scheme, where a $3 \times 3 \times 1$ gird was adopted in geometry optimizations and calculations of electronic properties. 59

The calculated STM images are obtained by using the Elastic Scattering Quantum Chemistry (ESQC) code. ${ }^{60}$ Here, the STM junction comprising the substrate, the adsorbates, the tip apex and the tip support is fully described at the atomic level. A set of semi-empirical extended Hückel orbitals is assigned on each atom site. The scattering of tunnel electrons trough the junction is calculated that allows the evaluation of the tunneling current with the Landauer formula. This technique has already proven its reliability with small ${ }^{61,62}$ and large ${ }^{63,64}$ molecular systems.

\section{ASSOCIATED CONTENT}

\section{Supporting Information}

The Supporting Information is available free of charge via the Internet at http://pubs.acs.org. 
The $\mathrm{dI} / \mathrm{dV}$ spectra for Kondo ON and OFF states with reference curves taken on the bare $\mathrm{Au}(111)$ surface; Fano fitting for Kondo resonance; Coexistence of the $\mathrm{G}_{3} \mathrm{Ni}_{3} \mathrm{Br}_{4}$ and $\mathrm{G}_{3} \mathrm{Ni}_{3} \mathrm{Br}_{3}$ motifs; The corresponding $\mathrm{dI} / \mathrm{dV}$ spectra on the coexistent motifs; Bonding manner between on-top Br and Ni trimer; PDOS of the $\mathrm{p}_{\mathrm{x}}, \mathrm{p}_{\mathrm{y}}$ and $\mathrm{p}_{\mathrm{z}}$ states of the top $\mathrm{Br}$ atom in a $\mathrm{G}_{3} \mathrm{Ni}_{3} \mathrm{Br}_{4}$ motif and differential charge density of the $\mathrm{G}_{3} \mathrm{Ni}_{3} \mathrm{Br}_{4}$ motif.

\section{AUTHOR INFORMATION}

\section{Corresponding Author}

*E-mail: xuwei@,tongji.edu.cn, ji.pascual@,nanogune.eu

\section{Author Contributions}

『 L.X. and H.L. contributed equally to this work.

The authors declare no competing financial interests.

\section{ACKNOWLEDGEMENTS}

W. Xu acknowledges financial support from the National Natural Science Foundation of China $(21622307,21790351)$ and the Fundamental Research Funds for the Central Universities. H. Lin acknowledges supports from the National Natural Science Foundation of China (21771134) and the Collaborative Innovation Center of Suzhou Nano Science \& Technology, the Priority Academic Program Development of Jiangsu Higher Education Institutions (PAPD), the 111 Project. JIP acknowledges financial support from the Spanish AEI (MAT2016-78293-C6 and the Maria de Maeztu Units of Excellence Program MDM-2016-0618).

\section{REFERENCES}

1. Bogani, L.; Wernsdorfer, W. Molecular Spintronics Using Single-Molecule Magnets. Nat. Mater. 2008, 7, 179-186.

2. Moth-Poulsen, K.; Bjørnholm, T. Molecular Electronics with Single Molecules in 
Solid-State Devices. Nat. Nanotech. 2009, 4, 551-556.

3. Rocha, A. R.; García-Suárez, V. M.; Bailey, S. W.; Lambert, C. J.; Ferrer, J.; Sanvito, S. Towards Molecular Spintronics. Nat. Mater. 2005, 4, 335-339.

4. Candini, A.; Klyatskaya, S.; Ruben, M.; Wernsdorfer, W.; Affronte, M. Graphene Spintronic Devices with Molecular Nanomagnets. Nano Lett. 2011, 11, 2634-2639.

5. Liu, L.; Yang, K.; Jiang, Y.; Song, B.; Xiao, W.; Li, L.; Zhou, H.; Wang, Y.; Du, S.; Ouyang, M. et al. Reversible Single Spin Control of Individual Magnetic Molecule by Hydrogen Atom Adsorption. Sci. Rep. 2013, 3, 1210.

6. Kondo, J. Resistance Minimum in Dilute Magnetic Alloys. Prog. Theor. Phys. 1964, 32, 37-49.

7. Kondo, J. Effect of Ordinary Scattering on Exchange Scattering from Magnetic Impurity in Metals. Phys. Rev. 1968, 169, 437-440.

8. Li, J.; Schneider, W.-D.; Berndt, R.; Delley, B. Kondo Scattering Observed at a Single Magnetic Impurity. Phys. Rev. Lett. 1998, 80, 2893-2896.

9. Madhavan, V.; Chen, W.; Jamneala, T.; Crommie, M. F.; Wingreen, N. S. Tunneling into a Single Magnetic Atom: Spectroscopic Evidence of the Kondo Resonance. Science 1998, 280, 567-569.

10. Houck, A. A.; Labaziewicz, J.; Chan, E. K.; Folk, J. A.; Chuang, I. L. Kondo Effect in Electromigrated Gold Break Junctions. Nano Lett. 2005, 5, 1685-1688.

11. Frisenda, R.; Gaudenzi, R.; Franco, C.; Mas-Torrent, M.; Rovira, C.; Veciana, J.; Alcon, I.; Bromley, S. T.; Burzurí, E.; van der Zant, H. S. J. Kondo Effect in a Neutral and Stable All Organic Radical Single Molecule Break Junction. Nano Lett. 2015, 15, 
3109-3114.

12. Kogan, A.; Amasha, S.; Kastner, M. A. Photon-Induced Kondo Satellites in a Single-Electron Transistor. Science 2004, 304, 1293-1295.

13. Goldhaber-Gordon, D.; Shtrikman, H.; Mahalu, D.; Abusch-Magder, D.; Meirav, U.; Kastner, M. A. Kondo Effect in a Single-Electron Transistor. Nature 1998, 391, 156-159.

14. Parks, J. J.; Champagne, A. R.; Costi, T. A.; Shum, W. W.; Pasupathy, A. N.; Neuscamman, E.; Flores-Torres, S.; Cornaglia, P. S.; Aligia, A. A.; Balseiro, C. A. et al. Mechanical Control of Spin States in Spin-1 Molecules and the Underscreened Kondo Effect. Science 2010, 328, 1370-1373.

15. Liang, W.; Shores, M. P.; Bockrath, M.; Long, J. R.; Park, H. Kondo Resonance in a Single-Molecule Transistor. Nature 2002, 417, 725-729.

16. Park, J.; Pasupathy, A. N.; Goldsmith, J. I.; Chang, C.; Yaish, Y.; Petta, J. R.; Rinkoski, M.; Sethna, J. P.; Abruña, H. D.; McEuen, P. L. et al. Coulomb Blockade and the Kondo Effect in Single-Atom Transistors. Nature 2002, 417, 722-725.

17. $\mathrm{Yu}, \mathrm{L} . \mathrm{H}$;; Natelson, D. The Kondo Effect in $\mathrm{C}_{60}$ Single-Molecule Transistors. Nano Lett. 2004, 4, 79-83.

18. Nygård, J.; Cobden, D. H.; Lindelof, P. E. Kondo Physics in Carbon Nanotubes. Nature 2000, 408, 342-346.

19. Cervetti, C.; Rettori, A.; Pini, M. G.; Cornia, A.; Repolles, A.; Luis, F.; Dressel, M.; Rauschenbach, S.; Kern, K.; Burghard, M. et al. The Classical and Quantum Dynamics of Molecular Spins on Graphene. Nat. Mater. 2016, 15, 164-168. 
20. Li, J.; Sanz, S.; Corso, M.; Choi, D. J.; Peña, D.; Frederiksen, T.; Pascual, J. I. Single Spin Localization and Manipulation in Graphene Open-Shell Nanostructures. Nat. commun. 2019, 10, 200.

21. Ternes, M.; Heinrich, A. J.; Schneider, W. D. Spectroscopic Manifestations of the Kondo Effect on Single Adatoms. J. Phys.-Condens. Mat. 2009, 21, 053001.

22. Heinrich, A. J.; Gupta, J. A.; Lutz, C. P.; Eigler, D. M. Single-Atom Spin-Flip Spectroscopy. Science 2004, 306, 466-469.

23. Baumann, S.; Paul, W.; Choi, T.; Lutz, C. P.; Ardavan, A.; Heinrich, A. J. Electron Paramagnetic Resonance of Individual Atoms on a Surface. Science 2015, 350, $417-$ 420.

24. Knorr, N.; Schneider, M. A.; Diekhöner, L.; Wahl, P.; Kern, K. Kondo Effect of Single Co Adatoms on Cu Surfaces. Phys. Rev. Lett. 2002, 88, 386-389.

25. Wahl, P.; Diekhöner, L.; Schneider, M. A.; Vitali, L.; Wittich, G.; Kern, K. Kondo Temperature of Magnetic Impurities at Surfaces. Phys. Rev. Lett. 2004, 93, 176603.

26. Schneider, M. A.; Vitali, L.; Wahl, P.; Knorr, N.; Diekhöner, L.; Wittich, G.; Vogelgesang, M.; Kern, K. Kondo state of Co impurities at noble metal surfaces. Appl. Phys. A 2005, 80, 937-941.

27. Schuh, T.; Miyamachi, T.; Gerstl, S.; Geilhufe, M.; Hoffmann, M.; Ostanin, S.; Hergert, W.; Ernst, A.; Wulfhekel, W. Magnetic Excitations of Rare Earth Atoms and Clusters on Metallic Surfaces. Nano Lett. 2012, 12, 4805-4809.

28. Otte, A. F.; Ternes, M.; von Bergmann, K.; Loth, S.; Brune, H.; Lutz, C. P.; Hirjibehedin, C. F.; Heinrich, A. J. The Role of Magnetic Anisotropy in the Kondo 
Effect. Nat. Phys. 2008, 4, 847-850.

29. Zhao, A.; Li, Q.; Chen, L.; Xiang, H.; Wang, W.; Pan, S.; Wang, B.; Xiao, X.; Yang, J.; Hou, J. G. et al. Controlling the Kondo Effect of an Adsorbed Magnetic Ion through Its Chemical Bonding. Science 2005, 309, 1542-1544.

30. Li, R.; Li, N.; Wang, H.; Weismann, A.; Zhang, Y.; Hou, S.; Wu, K.; Wang, Y. Tuning the Spin-Related Transport Properties of FePc on Au(111) through SingleMolecule Chemistry. Chem. Commun. 2018, 54, 9135-9138.

31. Heinrich, B. W.; Ahmadi, G.; Müller, V. L.; Braun, L.; Pascual, J. I.; Franke, K. J. Change of the Magnetic Coupling of a Metal-Organic Complex with the Substrate by a Stepwise Ligand Reaction. Nano Lett. 2013, 13, 4840-4843.

32. Li, J.; Merino-Díez, N.; Carbonell-Sanromà, E.; Vilas-Varela, M.; de Oteyza, D. G.; Peña, D.; Corso, M.; Pascual, J. I. Survival of Spin State in Magnetic Porphyrins Contacted by Graphene Nanoribbons. Sci. Adv. 2018, 4, eaaq0582.

33. Li, J.; Friedrich, N.; Merino, N.; de Oteyza, D. G.; Peña, D.; Jacob, D.; Pascual, J. I. Electrically Addressing the Spin of a Magnetic Porphyrin through Covalently Connected Graphene Electrodes. Nano Lett. 2019, 19, 3288-3294.

34. Heinrich, B. W.; Ehlert, C.; Hatter, N.; Braun, L.; Lotze, C.; Saalfrank, P.; Franke, K. J. Control of Oxidation and Spin State in a Single-Molecule Junction. ACS Nano 2018, 12, 3172-3177.

35. Iancu, V.; Deshpande, A.; Hla, S.-W. Manipulation of the Kondo Effect via TwoDimensional Molecular Assembly. Phys. Rev. Lett. 2006, 97, 266603.

36. Heinrich, B. W.; Braun, L.; Pascual, J. I.; Franke, K. J. Tuning the Magnetic 
Anisotropy of Single Molecules. Nano Lett. 2015, 15, 4024-4028.

37. Gao, L.; Ji, W.; Hu, Y. B.; Cheng, Z. H.; Deng, Z. T.; Liu, Q.; Jiang, N.; Lin, X.;

Guo, W.; Du, S. X. et al. Site-Specific Kondo Effect at Ambient Temperatures in IronBased Molecules. Phys. Rev. Lett. 2007, 99, 106402.

38. Minamitani, E.; Tsukahara, N.; Matsunaka, D.; Kim, Y.; Takagi, N.; Kawai, M. Symmetry-Driven Novel Kondo Effect in a Molecule. Phys. Rev. Lett. 2012, 109, 086602.

39. Franke, K. J.; Schulze, G.; Pascual, J. I. Competition of Superconducting Phenomena and Kondo Screening at the Nanoscale. Science 2011, 332, 940-944.

40. Girovsky, J.; Nowakowski, J.; Ali, M. E.; Baljozovic, M.; Rossmann, H. R.; Nijs, T.; Aeby, E. A.; Nowakowska, S.; Siewert, D.; Srivastava, G. et al. Long-Range Ferrimagnetic Order in a Two-Dimensional Supramolecular Kondo Lattice. Nat. Commun. 2017, 8, 15388.

41. Tsukahara, N.; Shiraki, S.; Itou, S.; Ohta, N.; Takagi, N.; Kawai, M. Evolution of Kondo Resonance from a Single Impurity Molecule to the Two-Dimensional Lattice. Phys. Rev. Lett. 2011, 106, 187201.

42. Gopakumar, T. G.; Tang, H.; Morillo, J.; Berndt, R. Transfer of Cl Ligands between AdsorbedIron Tetraphenylporphyrin Molecules. J. Am. Chem. Soc. 2012, 134, $11844-11847$.

43. Tsukahara, N.; Minamitani, E.; Kim, Y.; Kawai, M.; Takagi, N. Controlling Orbital-Selective Kondo Effects in a Single Molecule through Coordination Chemistry. J. Chem. Phys. 2014, 141, 054702. 
44. Stróżecka, A.; Soriano, M.; Pascual, J. I.; Palacios, J. J. Reversible Change of the Spin State in a Manganese Phthalocyanine by Coordination of CO Molecule. Phys. Rev. Lett. 2012, 109, 147202.

45. Wäckerlin, C.; Tarafder, K.; Girovsky, J.; Nowakowski, J.; Hählen, T.; Shchyrba, A.; Siewert, D.; Kleibert, A.; Nolting, F.; Oppeneer, P. M. et al. Ammonia Coordination Introducing a Magnetic Moment in an On-Surface Low-Spin Porphyrin. Angew. Chem. Int. Ed. 2013, 52, 4568-4571.

46. Krull, C.; Robles, R.; Mugarza, A.; Gambardella, P. Site- and Orbital-Dependent Charge Donation and Spin Manipulation in Electron-Doped Metal Phthalocyanines. Nat. Mater. 2013, 12, 337-343.

47. Xie, L.; Zhang, C.; Ding, Y.; Xu, W. Structural Transformation and Stabilization of Metal-Organic Motifs Induced by Halogen Doping. Angew. Chem. Int. Ed. 2017, 56, $5077-5081$.

48. Fano, U. Effects of Configuration Interaction on Intensities and Phase Shifts. Phys. Rev. 1961, 124, 1866-1878.

49. Nagaoka, K.; Jamneala, T.; Grobis, M.; Crommie, M. F. Temperature Dependence of a Single Kondo Impurity. Phys. Rev. Lett. 2002, 88, 077205.

50. Fernández-Torrente, I.; Franke, K. J.; Pascual, J. I., Vibrational Kondo Effect in Pure Organic Charge-Transfer Assemblies. Phys. Rev. Lett. 2008, 101, 217203.

51. Moro-Lagares, M.; Korytár, R.; Piantek, M.; Robles, R.; Lorente, N.; Pascual, J. I.; Ibarra, M. R.; Serrate, D. Real Space Manifestations of Coherent Screening in Atomic Scale Kondo Lattices. Nat. commun. 2019, 10, 2211. 
52. Besenbacher, F. Scanning Tunnelling Microscopy Studies of Metal Surfaces. Rep. Prog. Phys. 1996, 59, 1737-1802.

53. Lægsgaard, E.; Österlund, L.; Thostrup, P.; Rasmussen, P. B.; Stensgaard, I.; Besenbacher, F. A High-Pressure Scanning Tunneling Microscope. Rev. Sci. Instrum. 2001, 72, 3537-3542.

54. Ruby, M. SpectraFox: A Free Open-Source Data Management and Analysis Tool for Scanning Probe Microscopy and Spectroscopy. SoftwareX 2016, 5, 31-36.

55. Kresse, G.; Furthmuller, J. Efficient Iterative Schemes for Ab Initio Total-Energy Calculations Using a Plane-Wave Basis Set. Phys. Rev. B 1996, 54, 11169-11186.

56. Blöchl, P. E. Projector Augmented-Wave Method. Phys. Rev. B 1994, 50, 17953 17979.

57. Perdew, J. P.; Burke, K.; Ernzerhof, M. Generalized Gradient Approximation Made Simple. Phys. Rev. Lett. 1996, 77, 3865-3868.

58. Grimme, S.; Antony, J.; Ehrlich, S.; Krieg, H. A Consistent and Accurate Ab Initio Parametrization of Density Functional Dispersion Correction (DFT-D) for the 94 Elements H-Pu. J. Chem. Phys. 2010, 132, 154104.

59. Monkhorst, H. J.; Pack, J. D. Special Points for Brillouin-Zone Integrations. Phys. Rev. B 1976, 13, 5188-5192.

60. Sautet, P.; Joachim, C. Calculation of the Benzene on Rhodium Stm Images. Chem. Phys. Lett. 1991, 185, 23-30.

61. Villagómez, C. J.; Castanié, F.; Momblona, C.; Gauthier, S.; Zambelli, T.; Bouju, X. Adsorption of Single 1,8-Octanedithiol Molecules on $\mathrm{Cu}(100)$. Phys. Chem. Chem. 
Phys. 2016, 18, 27521-27528.

62. Yu, M.; Kalashnyk, N.; Barattin, R.; Benjalal, Y.; Hliwa, M.; Bouju, X.; Gourdon, A.; Joachim, C.; Lægsgaard, E.; Besenbacher, F. et al. Self-assembly of HydrogenBonded Chains of Molecular Landers. Chem. Commun. 2010, 46, 5545-5547.

63. Xu, W.; Dong, M. D.; Gersen, H.; Vázquez-Campos, S.; Bouju, X.; Lægsgaard, E.; Stensgaard, I.; Crego-Calama, M.; Reinhoudt, D. N.; Linderoth, T. R. et al. Exploring the Transferability of Large Supramolecular Assemblies to the Vacuum-Solid Interface. Nano Res. 2009, 2, 535-542.

64. Villagomez, C. J.; Guillermet, O.; Goudeau, S.; Ample, F.; Xu, H.; Coudret, C.; Bouju, X.; Zambelli, T.; Gauthier, S. Self-Assembly of Enantiopure Domains: The Case of Indigo on Cu(111). J. Chem. Phys. 2010, 132, 074705. 


\section{Table of Contents}
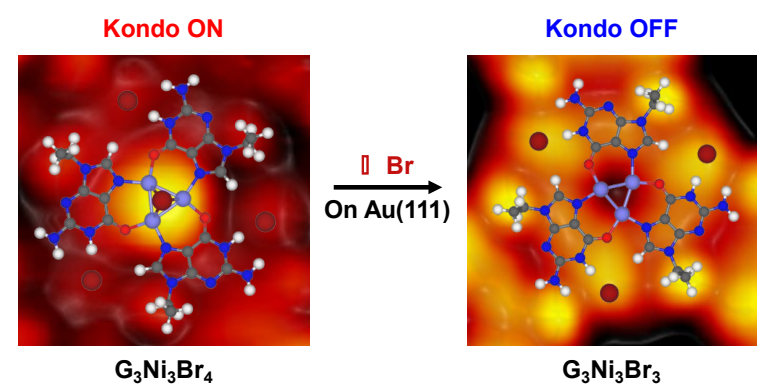

From the interplay of scanning tunneling microscopy/spectroscopy and density functional theory calculations, we successfully demonstrate that the spin of a Ni trimer within a metal-organic motif can be switched by controlling the attachment of on-top $\mathrm{Br}$ atom, which is monitored via Kondo effect. 